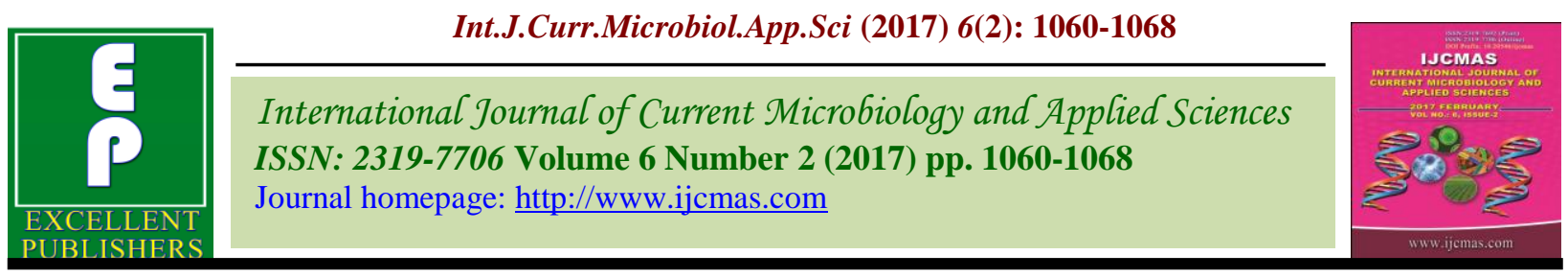

Original Research Article

http://dx.doi.org/10.20546/ijcmas.2017.602.119

\title{
Genetic Diversity Studies in Thermo Tolerant Genotypes of Tomato (Solanum lycopersicum L.)
}

\author{
Manish Kumar ${ }^{1}$, R.K. $\operatorname{Yadav}^{1}$, Manoj Kumar ${ }^{1}$ and Akshay Talukdar ${ }^{2}$ \\ ${ }^{1}$ Division of Vegetable Science, ICAR-Indian Agricultural Research Institute, \\ New Delhi-110012, India \\ ${ }^{2}$ Division of Genetics and Plant Breeding, ICAR-Indian Agricultural Research Institute, \\ New Delhi-110012, India \\ *Corresponding author
}

\section{A B S T R A C T}

\section{Keywords}

Solanum

lycopersicum,

Genetic diversity,

$\mathrm{D}^{2}$ analysis,

Clustering pattern.

Article Info

Accepted:

20 January 2017

Available Online:

10 February 2017
Genetic diversity study involving twenty one thermo tolerant tomato genotypes was carried out for twelve physiological and biochemical characters at Indian Agricultural Research Institute, New Delhi during summer season of the year 2014. All the genotypes were grouped into 4 distinct clusters using $\mathrm{D}^{2}$ statistics. Cluster I and cluster II comprised of 10 genotypes represented by heat tolerant genotypes while cluster III showed 4 heat sensitive genotypes. The clustering analysis of all genotypes showed that heat sensitive and heat tolerant genotypes were represented by distinct clusters. Maximum intra cluster distance was recorded in cluster IV (2.21), while the inter cluster distance was found maximum between cluster III and cluster IV (5.95). Cluster means of all genotypes showed that genotypes in cluster I exhibited minimum cluster mean value for chlorophyll b $(0.24$ $\mathrm{mg} / 100 \mathrm{~g})$ and maximum for proline content $(364 \mu \mathrm{g} / \mathrm{g})$. Principal component analysis showed that the first 4 PC's had proportionate contribution of $45.2 \%, 64.1 \%, 76.7 \%$ and $88.9 \%$ respectively.

\section{Introduction}

Tomato is an herbaceous plant belongs to Nightshade family. It is an important Solanaceous vegetable crop grown worldwide. The nutritional value of tomato makes it useful for reducing cardiovascular risk associated with type-II diabetes (Freedman et al., 2008). Tomato intake helps in weight loss and controlling obesity, eye disorders, night blindness, urinary tract infection and liver disorders. In any crop improvement programme, the proper selection of diverse parents is a prerequisite (Singh et al., 2002). The assessment of genetic diversity helps in differentiating well-defined population. Several methods have been proposed for diversity analysis; among them Mahalanobis generalized distance occupies a unique place and an efficient method to gauge the extent of diversity among genotypes, which quantify the differences among several quantitative traits. Therefore, the present investigation was undertaken involving various genotypes and popular varieties to assess the extent of genetic diversity in different thermo tolerant tomato genotypes. 


\section{Materials and Methods}

Genetic diversity analysis of twenty one thermo tolerant tomato genotypes was performed in the Research Farm of Division of Vegetable Science, Indian Agricultural Research Institute, New Delhi during the summer season (March-June) of the year 2014. The experimental material consisted of 21 contrasting thermo tolerant tomato genotypes (Table 1) and the experiment was laid out in randomized block design with 3 replications. The physiological characters viz., relative water content, membrane stability index and chlorophyll content $(\mathrm{a}, \mathrm{b}$ and total chlorophyll, chlorophyll a/b ratio) were analysed according to method of Brass and Weatherley (1962), Premachandra et al., (1990) and Hiscox and Israelstam (1979) respectively, whereas the biochemical parameters viz., TSS, lycopene (as described by Ranganna, 1977), ascorbic acid and acidity (as described in A.O.A.C., 1975) and proline (as described by Bates et al., 1973) were recorded for estimating the genetic diversity. Mahalanobis (1936) $\mathrm{D}^{2}$ analysis was used for assessing the genetic divergence among the test entries involving different characters. The genotypes were grouped into different clusters following Tocher's method as described by Rao (1952).

\section{Results and Discussion}

Twenty one thermo tolerant tomato genotypes were analysed for genetic divergence following Mahalanobis $\mathrm{D}^{2}$ statistical procedure. Considering physiological and biochemical characters, 21 tomato genotypes were categorized into distinct clusters and their Euclidian distance using $D^{2}$ statistics. 21 genotypes were grouped into 4 distinct clusters (Table 2). The grouping of genotypes into different clusters showed the presence of ample amount of genetic diversity among the genotypes under study. These results are in close conformation with the findings of Sharma et al., (2009), Basavaraj et al., (2010), Evgenidis et al., (2011), Iqbal et al., (2014) and Dar et al., (2015). Cluster I and cluster II comprised of 10 genotypes represented by heat tolerant genotypes while cluster III showed 4 heat sensitive genotypes namely, Pusa Ruby, Pusa 120, Pusa Rohini and Pusa Gaurav. All the wild genotypes and exotic collections were grouped into cluster IV. The clustering analysis of 21 genotypes showed that heat sensitive and heat tolerant genotypes were represented by distinct clusters.

Average intra (diagonal) and inter cluster $\left(\mathrm{D}^{2}\right)$ distance of different clusters (Table 3) showed that maximum intra cluster distance was recorded in cluster IV (2.21), while the inter cluster distance was found maximum between cluster III and cluster IV (5.95), followed by cluster II and cluster III (5.42). The maximum inter cluster distance showed the presence of greater divergence within the cluster. Similar findings were also reported by Sekhar et al., (2008) and Reddy et al., (2013) in tomato.

Cluster means of all genotypes (Table 4) revealed that genotypes in cluster I exhibited minimum cluster mean value for chlorophyll b $(0.24 \mathrm{mg} / 100 \mathrm{~g})$ and maximum for proline content $(364 \mu \mathrm{g} / \mathrm{g})$. The lines fall in to same cluster having lowest degree of divergence (Fig. 1). Similarly genotypes in cluster IV recorded maximum cluster mean for proline $(380 \mu \mathrm{g} / \mathrm{g})$. Similar trends were found for other clusters also. Low cluster mean value in all the clusters was recorded for acidity showed that there was least diversity among the 21 genotypes for this trait.

The Jaccard's similarity matrix was subjected to principal component analysis. PCA resolves complex relationships into interactions of fewer and simpler factors. First four PC's having eigen value greater than one 
accounting for $88.9 \%$ of the total variation (Table 5, Fig. 2). This finding has the similarity with the findings of Glogovac et al., (2012) based on biochemical fruit traits. The first 4 PC's had proportionate contribution of $45.2 \%, \quad 64.1 \%, \quad 76.7 \%$ and $88.9 \%$ respectively. PC-1 showed positive factor loading for RWC (0.37), Chlorophyll b (0.16), chlorophyll $\mathrm{a} / \mathrm{b}$ ratio $(0.0 .14)$, lycopene $(0.52)$, proline $(0.07)$ and fruit yield per plant (0.02). Contribution of lycopene was highest in PC-1 and indicated its role in total divergence. Similar result was also found by Lekshmi et al., (2016).

Thus the present experiment clearly revealed that 21 tomato genotypes were categorized into 4 distinct clusters using $\mathrm{D}^{2}$ statistics based on physiological and biochemical characters.

Table.1 List of genotypes and standard released varieties included in this study

\begin{tabular}{|c|c|c|}
\hline Sl. No. & Genotypes/Varieties & Tolerance level \\
\hline 1 & Pusa Sadabahar & $\mathrm{HT}$ \\
\hline 2 & Pusa Ruby & HS \\
\hline 3 & Pusa 120 & HS \\
\hline 4 & Pusa Rohini & HS \\
\hline 5 & Pusa Gaurav & HS \\
\hline 6 & Pusa Sheetal & $\mathrm{HT}$ \\
\hline 7 & Chikko & $\mathrm{HT}$ \\
\hline 8 & LP-2 & HT \\
\hline 9 & PSH-3 & HT \\
\hline 10 & TH-348-T2 & HT \\
\hline 11 & Balkan & HT \\
\hline 12 & TH-348-4-R & $\mathrm{HT}$ \\
\hline 13 & TH-348-4-2 & HT \\
\hline 14 & TH-348-4-5-1 & HT \\
\hline 15 & Spr-1* & $\mathrm{HT}$ \\
\hline 16 & Spr-2* & HT \\
\hline 17 & $\mathrm{Spm}^{* *}$ & $\mathrm{HT}$ \\
\hline 18 & SPM $1 * *$ & HT \\
\hline 19 & SPM 2** & HT \\
\hline 20 & SPM $3 * *$ & HT \\
\hline 21 & SPM 4** & HT \\
\hline
\end{tabular}

(Where, HT- heat tolerant, HS- heat sensitive) (Where, ${ }^{*}$ S. peruvianum, ${ }^{*} *$ S. pimpinellifolium) 
Table. 2 Group constellation of twenty one tomato genotypes based on Mahalanobis $\mathrm{D}^{2}$ statistics

\begin{tabular}{|c|c|l|}
\hline Clusters & No. of genotypes & \multicolumn{1}{c|}{ Name of genotypes } \\
\hline I & 2 & Chikko, PSH-3 \\
\hline II & 8 & $\begin{array}{l}\text { Pusa Sadabahar, Pusa Sheetal, LP-2, TH-348-T2, Balkan, TH-348-4- } \\
\text { R, TH-348-4-2, TH-348-4-5-1 }\end{array}$ \\
\hline III & 4 & Pusa Ruby, Pusa 120, Pusa Rohini, Pusa Gaurav \\
\hline IV & 7 & Spr1, Spr2, Spm, SPM1, SPM2, SPM3, SPM4 \\
\hline
\end{tabular}

Table.3 Estimates of average intra (diagonal) and inter cluster distance for different clusters for twenty one tomato genotypes

\begin{tabular}{|c|c|c|c|c|}
\hline Clusters & I & II & III & IV \\
\hline I & 1.434 & & & \\
\hline II & 3.375 & 2.084 & & \\
\hline III & 4.500 & 5.427 & 1.392 & \\
\hline IV & 4.584 & 3.168 & 5.951 & 2.217 \\
\hline
\end{tabular}

Table.4 Cluster means of physiological and biochemical traits of twenty one tomato genotypes under heat stress

\begin{tabular}{|c|c|c|c|c|c|c|c|c|c|}
\hline \multirow{2}{*}{ Sl. No. } & \multirow{2}{*}{$\begin{array}{c}\text { Cluster } \\
\text { Traits }\end{array}$} & \multicolumn{2}{|c|}{ I } & \multicolumn{2}{c|}{ II } & \multicolumn{3}{c|}{ III } & \multicolumn{3}{c|}{ IV } \\
\cline { 3 - 10 } & Mean & SE \pm & Mean & SE \pm & Mean & SE \pm & Mean & SE \pm \\
\hline 1 & RWC $(\%)$ & 75.9 & 3.21 & 76.94 & 3.13 & 66.38 & 2.22 & 77.66 & 3.52 \\
\hline 2 & MSI \% & 77.83 & 2.59 & 81.73 & 2.33 & 68.01 & 3.48 & 80.38 & 4.15 \\
\hline 3 & chl a $(\mathrm{mg} / \mathrm{g})$ & 0.92 & 0.23 & 1.31 & 0.2 & 1.02 & 0.25 & 1.45 & 0.25 \\
\hline 4 & chl b (mg/g) & 0.24 & 0.06 & 0.39 & 0.03 & 0.24 & 0.05 & 0.38 & 0.05 \\
\hline 5 & $\begin{array}{c}\text { Total chl } \\
(\mathrm{mg} / \mathrm{g})\end{array}$ & 1.16 & 0.29 & 1.7 & 0.2 & 1.27 & 0.3 & 1.82 & 0.27 \\
\hline 6 & chl a/b ratio & 3.89 & 0.06 & 3.41 & 0.59 & 4.2 & 0.3 & 3.91 & 0.71 \\
\hline 7 & TSS $\left({ }^{\circ}\right.$ Brix $)$ & 5.7 & 0.09 & 5.79 & 0.48 & 5.22 & 0.15 & 7.81 & 0.42 \\
\hline 8 & $\begin{array}{c}\text { Lycopene } \\
(\mathrm{mg} / 100 \mathrm{~g})\end{array}$ & 1.7 & 0.25 & 1.86 & 0.26 & 1.11 & 0.09 & 1.98 & 0.12 \\
\hline 9 & $\begin{array}{c}\text { Ascorbic } \\
\text { Acid } \\
(\mathrm{mg} / 100 \mathrm{~g})\end{array}$ & 16.85 & 2.33 & 16.82 & 3.58 & 17.23 & 1.46 & 21.5 & 1.41 \\
\hline 10 & Acidity $(\%)$ & 0.42 & 0.03 & 0.36 & 0.03 & 0.34 & 0.01 & 0.37 & 0.05 \\
\hline 11 & $\begin{array}{c}\text { Proline } \\
(\mu \mathrm{\mu g} / \mathrm{g})\end{array}$ & 364.34 & 10.36 & 361.6 & 27.66 & 276.02 & 8.15 & 380.34 & 24.88 \\
\hline 12 & $\begin{array}{c}\text { Yield/plant } \\
(\mathrm{g})\end{array}$ & 182.20 & 11.59 & 295.00 & 15.2 & 165.20 & 9.36 & 579.00 & 18.31 \\
\hline
\end{tabular}




\section{Int.J.Curr.Microbiol.App.Sci (2017) 6(2): 1060-1068}

Table.5 Principal component analysis for physiological and biochemical traits of twenty one tomato genotypes under heat stress

\begin{tabular}{|c|c|c|c|c|c|c|c|c|c|c|c|c|}
\hline Parameters & $\mathrm{PC} 1$ & $\mathrm{PC} 2$ & PC3 & PC4 & PC5 & PC6 & PC7 & PC8 & PC9 & PC10 & PC11 & $\mathrm{PC} 12$ \\
\hline Eigen value (root) & 5.42 & 2.27 & 1.50 & 1.48 & 0.47 & 0.37 & 0.19 & 0.15 & 0.11 & 0.02 & 0.02 & 0.00 \\
\hline Cumulative eigen value & 5.42 & 7.69 & 9.20 & 10.67 & 11.15 & 11.51 & 11.71 & 11.86 & 11.98 & 11.99 & 12.00 & 12.00 \\
\hline Explained variation $(\%)$ & 45.20 & 18.9 & 12.56 & 12.29 & 3.93 & 3.05 & 1.65 & 1.26 & 0.96 & 0.17 & 0.01 & 0.00 \\
\hline Cumulative explained variation (\%) & 45.20 & 64.1 & 76.69 & 88.98 & 92.91 & 95.96 & 97.61 & 98.87 & 99.83 & 100 & 100.01 & 100.01 \\
\hline \multicolumn{13}{|c|}{ Eigen vectors } \\
\hline & $\mathrm{PC} 1$ & $\mathrm{PC} 2$ & PC3 & PC4 & PC5 & PC6 & PC7 & PC8 & PC9 & $\mathrm{PC} 10$ & PC11 & PC12 \\
\hline RWC (\%) & 0.37 & 0.38 & 0.29 & 0.35 & 0.326 & -0.09 & 0.26 & 0.37 & 0.18 & 0.14 & 0.36 & 0.08 \\
\hline MSI \% & -0.19 & -0.19 & 0.28 & -0.03 & 0.226 & 0.38 & 0.33 & -0.13 & 0.41 & -0.10 & -0.05 & -0.59 \\
\hline chl a (mg/g) & -0.07 & 0.02 & -0.35 & 0.14 & -0.257 & -0.59 & 0.24 & -0.07 & 0.35 & -0.41 & 0.20 & -0.18 \\
\hline chl b (mg/g) & 0.16 & 0.06 & -0.33 & -0.42 & -0.373 & 0.12 & 0.28 & 0.22 & 0.17 & 0.58 & 0.12 & -0.16 \\
\hline Total chl (mg/g) & -0.18 & 0.26 & 0.03 & 0.02 & 0.028 & 0.08 & -0.52 & -0.36 & 0.61 & 0.29 & 0.16 & 0.15 \\
\hline chl a/b ratio & 0.14 & 0.16 & -0.02 & -0.33 & -0.089 & 0.39 & 0.08 & -0.28 & -0.11 & -0.42 & 0.61 & 0.18 \\
\hline $\operatorname{TSS}\left({ }^{\circ}\right.$ Brix $)$ & -0.61 & -0.30 & 0.07 & 0.10 & 0.084 & -0.09 & 0.38 & -0.08 & -0.09 & 0.29 & 0.33 & 0.38 \\
\hline Lycopene (mg/100g) & 0.52 & -0.64 & -0.03 & 0.05 & 0.007 & 0.04 & 0.07 & -0.11 & 0.35 & -0.02 & -0.09 & 0.40 \\
\hline Ascorbic Acid (mg/100g) & -0.29 & 0.24 & -0.02 & -0.19 & -0.057 & 0.21 & 0.15 & 0.43 & 0.35 & -0.33 & -0.36 & 0.45 \\
\hline Acidity (\%) & -0.14 & -0.40 & 0.07 & -0.16 & 0.025 & -0.04 & -0.47 & 0.59 & 0.07 & -0.12 & 0.39 & -0.16 \\
\hline Proline $(\mu \mathrm{g} / \mathrm{g})$ & 0.07 & 0.03 & 0.45 & -0.68 & 0.205 & -0.51 & 0.07 & -0.12 & 0.09 & 0.01 & -0.08 & 0.04 \\
\hline Yield/plant (g) & 0.02 & -0.01 & -0.63 & -0.18 & 0.759 & -0.06 & 0.01 & -0.02 & 0.01 & 0.02 & 0.01 & 0.01 \\
\hline
\end{tabular}


Figure.1 Dendrogram showing clustering pattern among twenty one genotypes of tomato

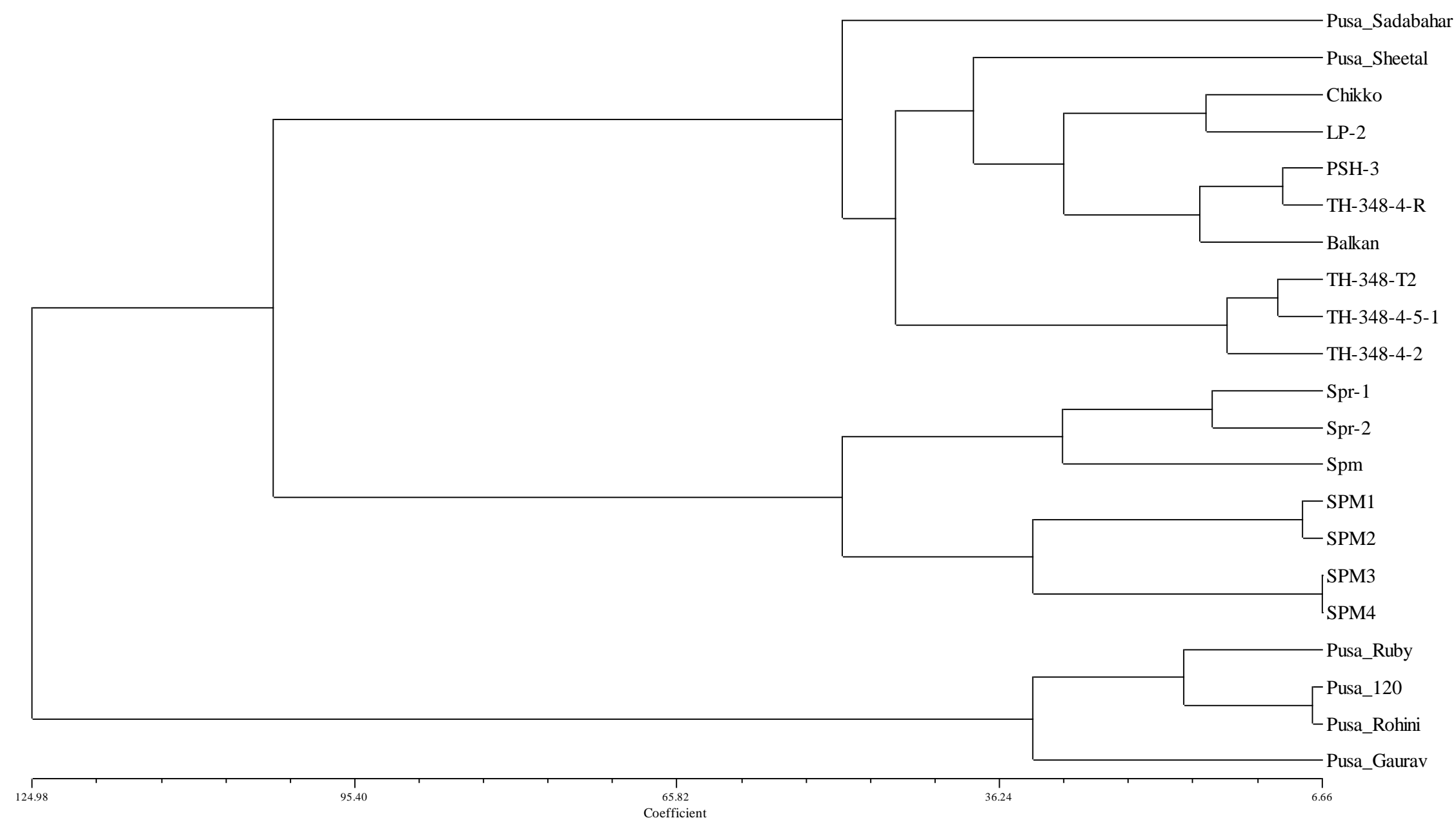




\section{Int.J.Curr.Microbiol.App.Sci (2017) 6(2): 1060-1068}

Figure.2 Principal component analysis of twenty one tomato genotypes revealed by physiological and biochemical traits

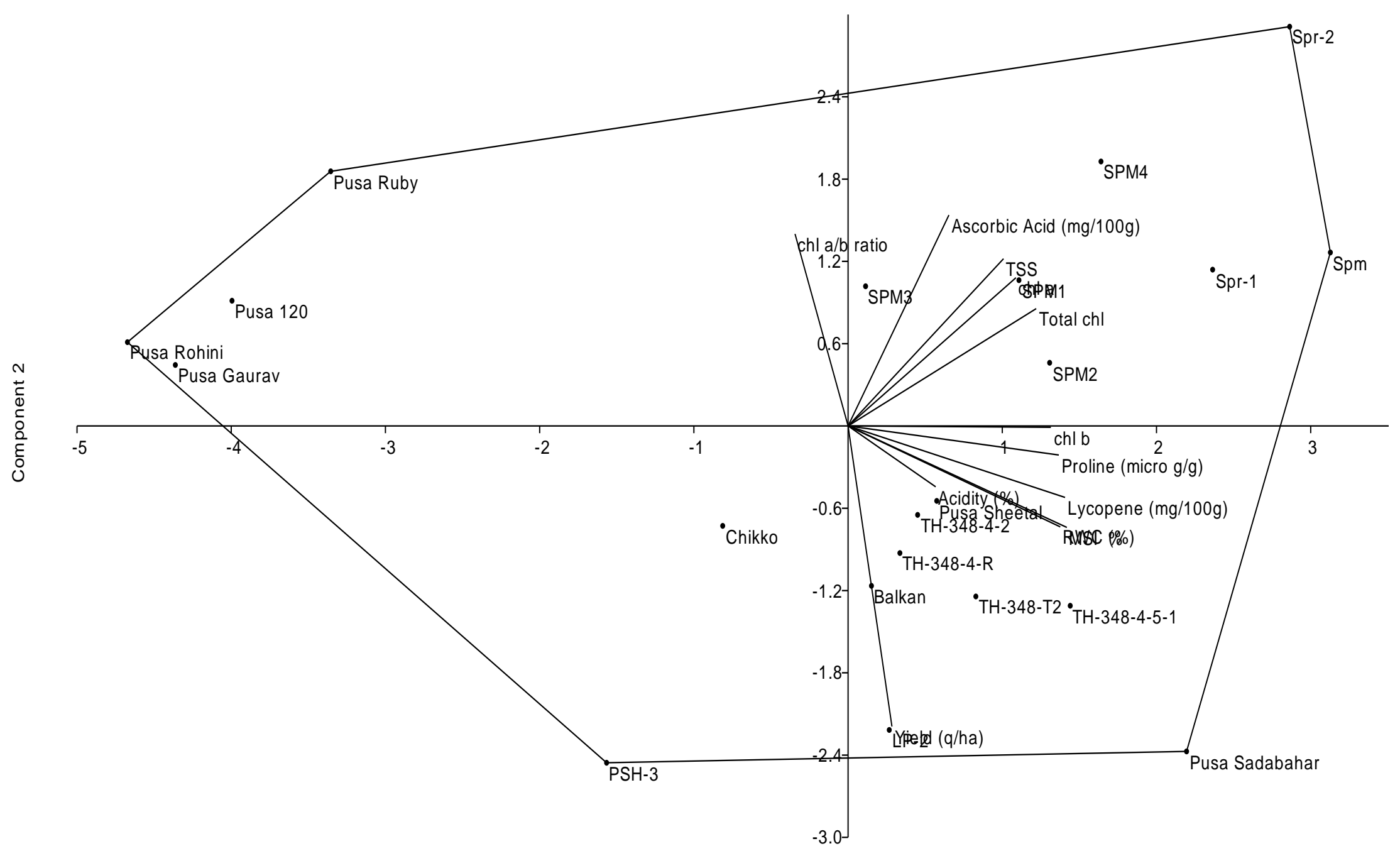

Component 1 
All the wild genotypes and exotic collections were grouped into two different clusters. 10 heat tolerant genotypes, namely Pusa Sadabahar, Pusa Sheetal, Chikko, LP-2, PSH3, TH-348-T2, Balkan, TH-348-4-R, TH-3484-2, TH-348-4-5-1 were represented by same cluster, while 4 heat sensitive genotypes, namely Pusa Ruby, Pusa 120, Pusa Rohini and Pusa Gaurav were grouped into another cluster. This showed that clustering was done effectively.

\section{Acknowledgement}

The author (Manish Kumar) is thankful to ICAR-Indian Agricultural Research Institute, New Delhi for the award of fellowship during the research programme.

\section{References}

A.O.A.C. 1975. In: Official Methods of Analysis. Publication of the Association of Analytical Chemists. Washington DC, USA. Pp. 401-404 and 829-831.

Basavaraj, N.S., Patil, B.C., Salimath, P.M., Hosamani, R.M., Krishnaraj, P.U. 2010. Genetic divergence in tomato (Solanum lycoperiscon [Mill.] Wettsd.). Karnataka J. Agri. Sci., 23(3): 508-539.

Bates, L.S., Waldren, R.P., Teare, I.D. 1973. Rapid determination of free proline for water stress studies. Plant and Soil, 39: 205-207.

Brass, H.D., Weatherley, P.E. 1962. A reexamination of the relative turgidity technique for estimating water deficits in leaves. Aus. J. Bio. Sci., 15: 143-28.

Dar, R.A., Sharma, J.P., Mushtaq, A. 2015. Genetic diversity among some productive genotypes of tomato (Lycopersicon esculentum Mill.). Afri. J. Biotechnol., 14(22): 1846-1853.

Evgenidis, G., Traka-Mavrona, E., KoutsikaSotiriou, M. 2011. Principal component and clusters analysis as a tool in the assessment of tomato hybrids and cultivars. Int. J. Agri., 6(23): 334-339.

Freedman, N.D., Park, Y., Subar, A.F., Hollenbeck, A.R., Leitzmann, M.F., Schatzkin, A., Abnet, C.C. 2008. Fruit and vegetable intake and head and neck cancer risk in a large United States prospective cohort study. Int. J. Cancer., 122(10): 2330-2336.

Glogovac, S., Takac, A., Tepic, A., Sumic, Z., Gvozdanovic-Varga, J., Cervenski, J., Popovic, V. 2012. Principal component analysis of tomato genotypes based on some morphological and biochemical quality indicators. Ratar. Povrt., 49(3): 296-301.

Hiscox, J.D., Israelstam, G.F. 1979. A method for extraction of chlorophyll from leaf tissue without maceration. Can. J. Bot., 57: 1332-1334.

Iqbal, Q., Saleem, M.Y., Hameed, A., Asghar, M. 2014. Assessment of genetic divergence in tomato through Agglomerative hierarchical clustering and Principal component analysis. Pakisthan J. Bot., 46(5): 1865-1870.

Lekshmi, S.L., Celine, V. A. 2016. Genetic Diversity Studies in Tomato (Solanum lycopersicum L.) Under Protected Conditions. Int. J. Curr. Microbiol. App. Sci., 5(4): 212-217.

Mahalanobis, P.C. 1936. On the generalized distance in statistics. Proceedings of National Academic Science (India). 2: 79-85.

Premachandra, G.S., Saneoka, H., Ogata, S. 1990. Cell membrane stability an indicator of drought tolerance as affected by applied $\mathrm{N}$ in soybean. $J$. Agri. Sci., 115: 63-66.

Ranganna, S. 1977. In: Manual for Analysis of Fruit and Vegetable Products. Tata McGraw Hill Publ. Co. Ltd., New Delhi. Pp. 634.

Rao, C.R. 1952. Advanced Statistical Methods in Biometrics Research. John 
Wiley and Sons, New York. Pp. 357369.

Reddy, B.R., Reddy, D.S., Reddaiah, K., Sunil, N. 2013. Studies on genetic variability, heritability and genetic advance for yield and quality traits in Tomato (Solanum lycopersicum L.). Int. J. Curr. Microbiol. Appl. Sci., 2(9): 238-244.

Sekhar, L., Prakash, B.G., Salimath, P.M., Sridevi, O., Patil, A.A. 2008. Genetic diversity among some productive hybrids of tomato (Lycopersicon esculentum Mill.). Karnataka J. Agri. Sci., 21(2): 264-265.

Sharma, J.P., Singh, A.K., Satesh, K., Sanjeev, K. 2009. Identification of traits for ideotype selection in tomato. Mysore J. Agri. Sci., 43: 222-226.

Singh, J.K., Singh, J.P., Jain, S.K., Joshi, A. 2002. Studies on genetic variability and its importance in tomato (Lycopesicum esculentum Mill.). Prog. Hort., 34: 7779.

\section{How to cite this article:}

Manish Kumar, R.K. Yadav, Manoj Kumar and Akshay Talukdar. 2017. Genetic Diversity Studies in Thermo Tolerant Genotypes of Tomato (Solanum lycopersicum L.). Int.J.Curr.Microbiol.App.Sci. $6(2)$ : 1060-1068.

doi:

http://dx.doi.org/10.20546/ijcmas.2017.602.119 